\title{
Ciclo de Mejora en el Aula en la asignatura de Matemáticas Específicas para Maestros en tiempos de COVID
}

\section{Classroom Improvement Cycle in the subject Specific Mathematics for Teachers in time of COVID}

ANA ESCUDERO-DOMÍNGUEZ

https://orcid.org/0000-0002-1620-3852

Universidad de Sevilla

Departamento de Didáctica de la Matemática

aescudero1@us.es

DOI: http://dx.doi.org/10.12795/9788447231003.085

Pp.: 1835-1854 


\section{Contexto de la asignatura}

Este ciclo de mejora en el aula (en adelante, CIMA) es la continuación de otras experiencias de innovación que hemos realizado durante cursos anteriores. Aunque este año, de nuevo, cambio de asignatura en la aplicación del CIMA, puedo aprovechar la experiencia en los mismos para esta nueva asignatura. Matemáticas Específicas para Maestros es una asignatura del primer curso del Grado de Primaria dentro del departamento de Didáctica de las Matemáticas. En esta los estudiantes se enfrentan por primera vez en el Grado a algunas nociones matemáticas, con el fin de aprender, conectar y comunicar ideas matemáticas que capaciten en el desempeño de la actividad profesional específica. Los alumnos cursan la asignatura en 2 días semanales, con una parte teórica de $2 \mathrm{~h}$ de duración y una práctica con una duración de 1 h (pues están divididos los alumnos en grupos más reducidos), es decir, 3h a la semana. La duración de este CIMA es de 30 horas, es decir, 10 semanas, abarcando desde el inicio de las clases hasta el 11 de diciembre de 2020. Esta materia está dividida en distintos bloques de contenidos, de los que voy a realizar mi CIMA atendiendo a los tres primeros: Sistemas de Numeración, Divisibilidad y Fracciones. El número total de alumnos matriculados en la misma es de 67 personas.

El año 2020 está siendo algo distinto por la evolución del virus COVID-19. El curso 2019-20 ha presentado un periodo de suspensión de clases presenciales y ante el nuevo curso, 2020-21, nos encontramos aún con incertidumbre sobre la presencialidad en las aulas. Esto ha hecho que nos planteemos nuevos retos en nuestra docencia. Comenzamos el 5 de octubre con una presencia menor en las aulas, de un tercio del grupo total, para respetar los horarios originales del Centro y atendiendo a la capacidad COVID-19 de los espacios, pero a finales de ese mismo mes, hemos pasado, de nuevo, a la suspensión de clases presenciales (en el caso de mi asignatura).

Ciclos de Mejora en el Aula (2020). Experiencias de Innovación Docente de la US Esta obra se distribuye con la licencia Creative Commons 
Esta asignatura fue una de las primeras que impartí cuando comencé a formar parte del Departamento de Didácticas de las Matemáticas y recuerdo como los alumnos eran bastante reacios a ella, pues casi todos la recuerdan como esa asignatura pendiente. De forma general, podemos decir que las clases de esta asignatura están planteadas con una exposición teórica mediante una presentación, seguida de unos ejercicios prácticos. Los contenidos de la asignatura son fundamentalmente conceptuales (45\%) y procedimentales (45\%), con menos de un $10 \%$ de actitudinales. Los contenidos son comunes a todos los grupos de la misma asignatura. Cada profesor personaliza las presentaciones, los ejercicios y las prácticas, su forma de dar las clases, pero todos seguimos una línea común. El examen final es común para todos, aunque la evaluación continua depende de cada profesor.

Las dos horas teóricas suelen estar preparadas para una exposición teórica, mediante presentación PowerPoint que se deja en la Enseñanza Virtual (en adelante EV) antes de cada clase, con lo que el alumno la tiene disponible previamente. En la hora semanal de prácticas se propone la resolución de problemas que se han dejado previamente en la plataforma. Con esta nueva situación a la que nos enfrentamos por la pandemia, en el caso de que sigamos la semipresencialidad preparada, en la que una parte de la clase esté en el aula física y otra parte en sesión virtual, la solución es que todos los estudiantes pasen al aula virtual, de modo que, independientemente de donde estén (en clase o en casa), todos trabajen en las mismas condiciones. Esto implica que los que vayan a clase lleven siempre su ordenador, creando un aula virtual. Esto puede desembocar en una asistencia virtual de todo el grupo, pero ya se irá viendo, pues entiendo que algunos alumnos les darán prioridad a la presencialidad de las clases prácticas.

Ciclos de Mejora en el Aula (2020). Experiencias de Innovación Docente de la US Esta obra se distribuye con la licencia Creative Commons 
Como herramientas para realizar las clases virtuales utilizaremos las distintas opciones que nos permite la EV, de entre hay que destacar el uso de la herramienta Blackboard Collaborate Ultra (en adelante BCU) con la que realizar videoconferencia. En la parte práctica donde el alumno debe resolver el problema de forma tutelada y yo voy atendiendo las dudas que van surgiendo al ir avanzando en la resolución, de forma individual primero, a veces en parejas -según la forma de trabajar del alumno- y luego de forma colectiva en la pizarra, la EV permite una opción en la que se pueden crear grupos, incluso éstos pueden aislarse, dejando de estar presente en la sesión virtual y volver a ella en cualquier momento, por lo que puede ser útil para estas sesiones.

\section{Diseño del CIMA}

Como hemos dicho anteriormente, nuestro CIMA abordará los tres primeros temas de la asignatura: Sistemas de Numeración, Divisibilidad y Fracciones. Cada uno de ellos tiene una duración aproximada de tres semanas. En el CIMA del curso pasado (Escudero-Domínguez, 2019) noté que, en la puesta en práctica, me costó muchísimo delimitarme a los tiempos establecidos, por ello este curso, ya desde el diseño hemos tenido en cuenta esta dificultad, siendo algo más flexible en ellos, por lo que voy a dejar como una semana de colchón, probablemente para poder explicar a los estudiantes la metodología a seguir. La experiencia en mis anteriores CIMA hace que sea consciente de mi dificultad a la hora de plantear mis mapas de contenidos, por lo que voy a intentar volcarme en ello. Otra novedad a introducir en este CIMA es el mostrar-modificar los mapas de contenidos con los estudiantes, pues así creo que ellos se sentirán más partícipes de la asignatura y a su vez esto puede facilitar el proceso de realización de la innovación docente.

Ciclos de Mejora en el Aula (2020). Experiencias de Innovación Docente de la US Esta obra se distribuye con la licencia Creative Commons 


\section{Principios didácticos}

Con el objetivo de abordar las principales dificultades detectadas, se ha diseñado un CIMA basado en una serie de principios didácticos que buscan la mejora de la atención de los estudiantes y la motivación e implicación de estos. Las innovaciones docentes en la materia están encaminadas hacia la implicación activa de los estudiantes en el proceso de enseñanza-aprendizaje, proponiendo que sean los discentes los que construyan el conocimiento (Carrillo, Climent, Gorgorió, Prat y Rojas, 2008). El nuevo modelo metodológico debe contener un mayor compromiso del alumno (Bain, 2007), menos exposición teórica por parte mía y más aplicación directa a problemas reales. Como ya intenté llevar a cabo en el anterior CIMA (Escudero-Domínguez, 2019), voy a intentar poner en práctica lo que Finkel (2008) llama "dar clase con la boca cerrada" donde debo intentar mantenerme en un segundo plano que empuje a los discentes a ser protagonistas de las clases.

\section{Modelo metodológico}

Tal y como en el CIMA anterior, pretendo seguir el siguiente modelo metodológico:

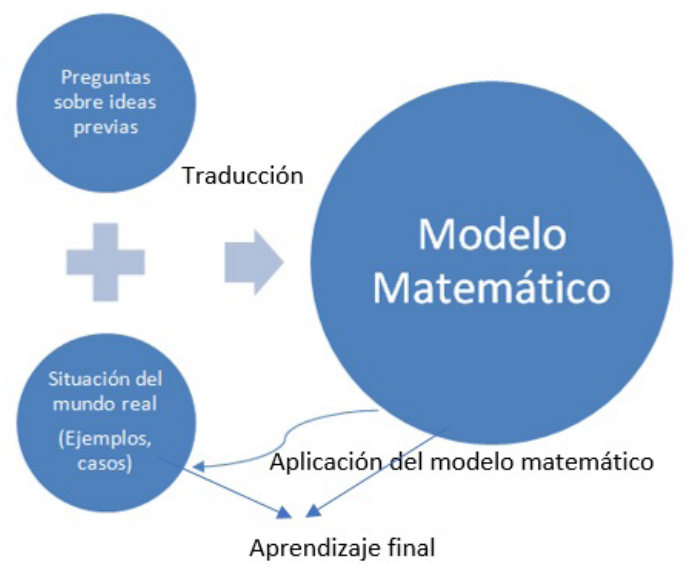

Figura 1. Modelo metodológico llevado a cabo

Ciclos de Mejora en el Aula (2020). Experiencias de Innovación Docente de la US Esta obra se distribuye con la licencia Creative Commons 
Por lo que la forma de trabajo que se propone es comenzar cada bloque de contenidos realizando preguntas a los estudiantes sobre sus ideas previas para conocer los conocimientos de los que partir. A continuación, se les pide que vayan buscando respuesta a las distintas preguntas, situaciones técnicas y/o ejercicios que se les plantea, ya sea mediante lecturas o material audiovisual. Esto lo pueden realizar de forma individual o en pareja. Todo ello pensado para ilustrar aplicaciones prácticas y fomentar la discusión. A partir de lo anterior, entre todos, pretendemos producir una translación al modelo teórico, generando unas conclusiones que nos conduzca al aprendizaje final. Este proceso metodológico invita al alumnado a la investigación y el razonamiento crítico y deductivo, basado en la resolución de problemas de distinto carácter que los discentes deben resolver de forma autónoma.

Mi modelo metodológico ideal es parecido al que trabaja Tous (2019) en el que los alumnos, antes de la clase, se miran lo que se va a trabajar, anotando sus dudas para poder ser resueltas durante la sesión. Una vez en clase los alumnos trabajan por grupo y van exponiendo por grupos sus conocimientos. Los demás alumnos podrán aportar información de la que no se haya hablado en la exposición o plantear dudas que no se hayan resuelto. Esto se llevará a cabo para cada parte del módulo que se está trabajando. Esta idea me gusta ya que pienso que, con la instrucción entre pares, los estudiantes hablan, trabajan y se enseñan unos a otros, eliminando la competitividad. Y se puede llevar a cabo mediante las distintas opciones que nos proporciona la EV.

Ciclos de Mejora en el Aula (2020). Experiencias de Innovación Docente de la US Esta obra se distribuye con la licencia Creative Commons 


\section{Mapa de contenidos}

Como hemos comentado anteriormente en esta asignatura priman los contenidos conceptuales y procedimentales. El primer paso para realizar un mapa de contenidos es ordenar y jerarquizar los contenidos. También tenemos que ver las relaciones entre estos e identificar aquellos que funcionan como articuladores.

Basado en los anteriores CIMA (Escudero-Domínguez, 2019,2018 ) mostramos un esquema general a tener presente durante toda la asignatura y que trabajaremos especialmente en su comienzo, abarcando las siguientes preguntas:

¿Por qué enseñar

matemáticas en el

Grado Ed. Primaria?
¿Qué matemáticas

enseñar?
¿Cómo las enseño?

Figura 2. Preguntas generales a tener presente durante toda la asignatura

Hemos detallado el mapa de contenidos del tema Fracciones (Figura 3). En él hemos diferenciado contenidos conceptuales (azul), procedimentales (celeste) y actitudinales (amarillo), mostrando la interacción entre los distintos tipos de contenidos. En esta figura queda patente lo comentado anteriormente sobre la asignatura, el gran peso que tienen los contenidos conceptuales y procedimentales, donde se pretende llevar al alumnado a trabajar las matemáticas directamente a través de problemas que los ayudan a entender la teoría, como se muestra más detalladamente en la secuencia de actividades.

Ciclos de Mejora en el Aula (2020). Experiencias de Innovación Docente de la US Esta obra se distribuye con la licencia Creative Commons 


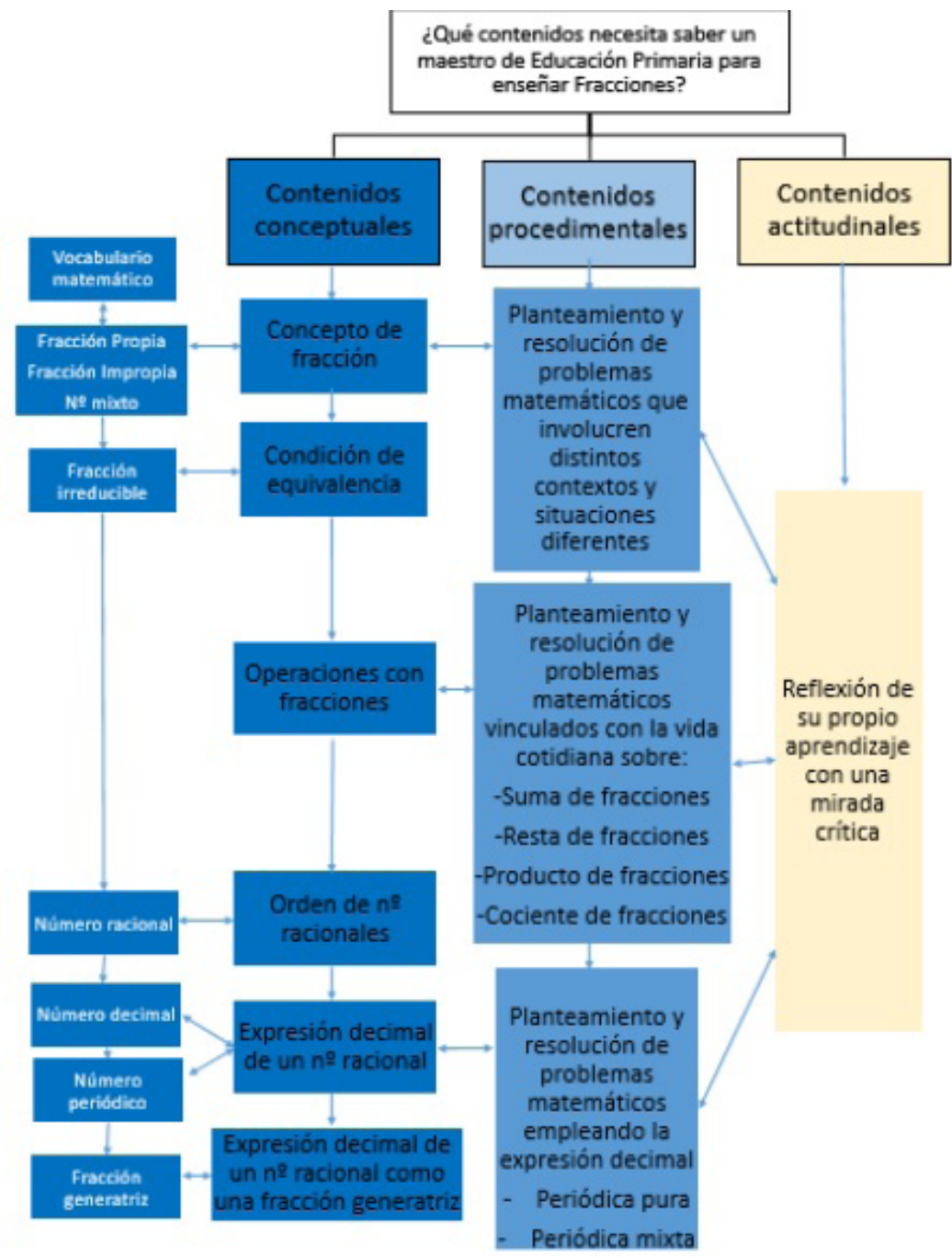

Figura 3. Mapa de contenidos del tema Fracciones

Ciclos de Mejora en el Aula (2020). Experiencias de Innovación Docente de la US (c) (i) $\odot$ Esta obra se distribuye con la licencia Creative Commons Reconocimiento-NoComercial-SinObraDerivada Internacional (CC BY-NC-ND 4.0.) 


\section{Secuencia de actividades}

Nos proponemos que esta no sea una asignatura memorística, sino que sean capaces de llevar a la práctica los conocimientos adquiridos, es decir, que sean capaces de resolver un problema que no hayan visto antes. Que mi rol sea el de orientar a los alumnos, ayudarlos a mejorar. ¿Cómo llevarlo a cabo? Pienso que para que los estudiantes aprendan no debe ser el profesor el que resuelva los ejercicios, sino los alumnos los que se enfrenten a esa resolución bajo la guía del profesor. Esto hace que se centren en la comprensión más que en la memorización, con lo que se facilita la retención del conocimiento a medio y largo plazo. Mi intención es enseñar a la gente a pensar, no a memorizar, además en su futuro es lo que van a tener que realizar, enseñar a sus estudiantes a pensar, a comprender los conceptos.

Como hemos comentado anteriormente, haremos uso en todo momento de la EV, creando un aula virtual, para que estén en igualdad de condiciones, tanto si estás en casa como en clase.

Ciclos de Mejora en el Aula (2020). Experiencias de Innovación Docente de la US Esta obra se distribuye con la licencia Creative Commons 
Tabla 1. Secuencia de actividades

\begin{tabular}{|l|l|}
\hline SESIÓN & \multicolumn{1}{c|}{ ACTIVIDADES } \\
\hline 1(2h) & $\begin{array}{l}\text { Se explica a los estudiantes la asignatura, distintos temas a tratar, } \\
\text { y que en este caso ellos son los responsables del aprendizaje, } \\
\text { mediante el desarrollo de distintas actividades guiadas por el } \\
\text { profesor. } \\
\text { En primer lugar, como docente debo de conocer las ideas previas } \\
\text { de los estudiantes. Para ello se le hace entrega de un cuestionario } \\
\text { inicial que deben completar, justificando su respuesta. } \\
\text { Cuestionario }\end{array}$ \\
$\begin{array}{ll}\text { 1. ¿Por qué enseñar matemáticas en el Grado Ed. Primaria? } \\
\text { 2. ¿Qué matemáticas enseñar? } \\
\text { 3. ¿Cómo enseñar matemáticas? } \\
\text { 4. ¿En qué consiste los sistemas de numeración? ¿Conoces al- } \\
\text { guna cultura que lo usara? } \\
\text { 5. Reflexiona sobre nuestros números ¿Se repiten?, ¿Podemos } \\
\text { contar a saltos?, ¿De cuánto en cuánto? } \\
\text { 6. Define múltiplo. Define divisor. } \\
\text { 7. ¿Qué es un número primo?, ¿Cuáles conoces? } \\
\text { 8. Definición de máximo común divisor (mcd). Definición de mí- } \\
\text { nimo común múltiplo (mcm). ¿Existe relación entre mcd y } \\
\text { mcm? } \\
\text { 9. Explica con tus palabras el concepto de fracción. ¿Se puede } \\
\text { reducir una fracción? } \\
\text { 10.Diferencia entre número racional, número decimal y número } \\
\text { periódico }\end{array}$ \\
$\begin{array}{l}\text { Presentamos el primer módulo a trabajar (Sistemas de } \\
\text { Numeración). Damos comienzo al tema haciendo entrega de un } \\
\text { documento de lectura con ejemplos de sistemas de numeración } \\
\text { aditivos y posicionales de algunas culturas. } \\
\text { Se da tiempo para que los alumnos reflexionen y se les pregunta } \\
\text { si con ello pueden responder a alguna de las preguntas del } \\
\text { cuestionario inicial (pregunta 4) y lanzamos algunas cuestiones } \\
\text { más que ayuden a aclarar los conceptos. } \\
\text { Se genera un debate en el que los alumnos expongan sus } \\
\text { conclusiones y entre todos llegara mostrar los distintos sistemas } \\
\text { de numeración de distintas culturas. }\end{array}$ \\
\hline
\end{tabular}

Ciclos de Mejora en el Aula (2020). Experiencias de Innovación Docente de la US Esta obra se distribuye con la licencia Creative Commons Reconocimiento-NoComercial-SinObraDerivada Internacional (CC BY-NC-ND 4.0.) 

la sesión. Se muestra a los alumnos el mapa de contenidos, explicando las tres ideas fundamentales que articulan nuestro sistema de numeración (no es aditivo, es decimal y posicional). Antes de las sesiones de la segunda semana, el profesor ha mirado los cuestionarios iniciales de los estudiantes para detectar si existe la necesidad de modificar el mapa conceptual y/o el modelo metodológico propuesto.

$3(2 \mathrm{~h}) \quad$ En esta sesión comenzamos con un repaso de lo aprendido en la anterior semana. Retomamos las tres ideas fundamentales en un sistema de numeración y se plantea una actividad.

Tras esta actividad, planteamos algunas actividades con las que los alumnos se enfrenten al cambio de base, comenzando por pasar de una base decimal a otra base (con el método de las divisiones sucesivas), luego dando paso a actividades donde tengan que pasar de una base distinta de 10 a base decimal (con el método de descomposición polinómica) y, por último, con actividades donde pasar un número de una base distinta de 10 a otra base distinta de 10 .

En esta sesión comenzamos repasando y desarrollando un esquema de los distintos cambios de base que vimos en la anterior clase. A continuación, desarrollaremos algunos ejercicios para afianzar contenidos.

Como hemos indicado, la dinámica será siempre la misma, mediante la EV, con la herramienta BCU los alumnos irán enfrentándose a la resolución de cada uno de los ejercicios, estando yo presente para resolver sus dudas, logrando que todos lleguen a la resolución, finalizando con la resolucióncomprobación del resultado.

Comenzamos planteando la siguiente pregunta: ¿Se podrán realizar las 4 operaciones aritméticas básicas con números en base distinta a la decimal?

Ante esta cuestión, abrimos el debate y poco a poco vamos trabajando el algoritmo de la suma, de la resta, de la multiplicación y de la división mediante ejercicios. En la resta repasamos el método de tomar prestado, de las llevadas y del ABN. En la multiplicación aparte del algoritmo actual, el algoritmo en celosía y el egipcio de duplicación. Y, por último, la división con el algoritmo expandido, mostrando si da tiempo el método ABN. 


\begin{tabular}{|c|c|}
\hline $6(1 h)$ & $\begin{array}{l}\text { En esta hora plantearemos un popurri de ejercicios para resolver } \\
\text { mediante las } 4 \text { operaciones aritméticas básicas en base distinta } \\
\text { a la decimal y se dejan más ejercicios para practicar en casa. }\end{array}$ \\
\hline $7(2 h)$ & $\begin{array}{l}\text { En esta primera parte de la sesión ultimamos el primer tema } \\
\text { corrigiendo y solventando dudas, exponiendo nuevamente el } \\
\text { mapa de contenidos. } \\
\text { En la segunda parte comenzamos el segundo módulo, es decir, } \\
\text { empezamos la divisibilidad. Esto lo realizamos partiendo de la } \\
\text { pregunta } 6 \text { del cuestionario inicial, es decir, haciendo una puesta } \\
\text { en común en gran grupo de lo que entienden por múltiplo y } \\
\text { divisor. Seguidamente intentaremos ir sacando las propiedades } \\
\text { de los divisores, para finalizar la sesión con la pregunta } 7 \text {. De } \\
\text { nuevo, en gran grupo mediante una puesta en común iremos } \\
\text { poniendo en la pizarra (BCU), todos los números primos que } \\
\text { conozcan, justificando su respuesta. A partir de ahí, el profesor } \\
\text { reformula las ideas extraídas del debate, y colocando en el otro } \\
\text { lado de la pizarra todos los números, intentamos hallar entre } \\
\text { todos los números primos del } 1 \text { al 120, es decir, llevar a cabo la } \\
\text { criba de Eratóstenes. }\end{array}$ \\
\hline $8(1 h)$ & $\begin{array}{l}\text { En esta sesión vamos a comenzar repasando lo anterior, mediante } \\
\text { la reflexión acerca de la factorización de un número, mostrando } \\
\text { el mapa de contenidos del tema, explicando los principios que } \\
\text { lo articulan. Se trata de hacer a los alumnos partícipes del mapa } \\
\text { de contenidos desarrollado, para que comprendan la jerarquía } \\
\text { de los conceptos básicos trabajados en el tema. }\end{array}$ \\
\hline $9(2 h)$ & $\begin{array}{l}\text { En esta sesión iremos viendo entre todos los criterios de } \\
\text { divisibilidad (del } 2 \text {, del } 3 \text {, del } 5 \text { y del 11). En la otra mitad de } \\
\text { la clase trabajaremos los divisores y conjunto de divisores de } \\
\text { un número mediante la deducción sistemática, planteando } \\
\text { ejercicios. } \\
\text { Aprovechando estos ejercicios haremos ver que estos números } \\
\text { poseen muchos divisores (p.e.: } 180 \text { es divisible por } 1,2,3 \text {, } \\
4,5,6,9, \ldots \text { ) así que necesitamos seguir algún método para } \\
\text { encontrarlos todos. Esta reflexión la realizaremos en gran grupo } \\
\text { e intentaremos encontrar el método para encontrarlos todos. }\end{array}$ \\
\hline $10(1 \mathrm{~h})$ & $\begin{array}{l}\text { En esta sesión haremos un repaso de la anterior y trabajaremos } \\
\text { con los criterios de divisibilidad, mediante ejercicios. }\end{array}$ \\
\hline
\end{tabular}

Ciclos de Mejora en el Aula (2020). Experiencias de Innovación Docente de la US Esta obra se distribuye con la licencia Creative Commons Reconocimiento-NoComercial-SinObraDerivada Internacional (CC BY-NC-ND 4.0.) 


\begin{tabular}{|c|c|}
\hline $11(2 h)$ & $\begin{array}{l}\text { En esta sesión vamos a repasar lo anterior y a comenzar a } \\
\text { trabajar la pregunta } 8 \text { del cuestionario inicial, en la que vamos } \\
\text { a pedir que la definición de máximo común divisor (mcd), } \\
\text { la definición de mínimo común múltiplo (mcm), así como si } \\
\text { existe relación entre ellos. Esto se realiza de forma práctica, a } \\
\text { partir de ejercicios. Una vez tengamos la definición de ambos, } \\
\text { ahondaremos sobre las propiedades y los métodos alternativos } \\
\text { para calcular el mcd. }\end{array}$ \\
\hline $12(1 \mathrm{~h})$ & $\begin{array}{l}\text { En esta sesión haremos un repaso de lo anterior y proponemos } \\
\text { ejercicios para practicar. }\end{array}$ \\
\hline $13(2 \mathrm{~h})$ & $\begin{array}{l}\text { En la primera parte de la sesión ultimamos el segundo tema } \\
\text { corrigiendo y solventando dudas, exponiendo nuevamente el } \\
\text { mapa de contenidos. } \\
\text { En la segunda parte comenzamos el tercer módulo, es decir, } \\
\text { empezamos las fracciones. Esto lo realizamos partiendo de las } \\
\text { preguntas } 9 \text { y } 10 \text { del cuestionario inicial, es decir, haciendo una } \\
\text { puesta en común en gran grupo de lo que entienden por fracción. } \\
\text { Tras las ideas de los alumnos vamos haciendo preguntas como: } \\
\text { ¿Se puede reducir una fracción? Para ir orientando la sesión } \\
\text { hacia lo que nosotros queremos que se vaya desarrollando. }\end{array}$ \\
\hline $14(1 \mathrm{~h})$ & $\begin{array}{l}\text { En esta sesión vamos a comenzar repasando lo anterior, } \\
\text { mediante la reflexión acerca del vocabulario recopilado en la } \\
\text { clase anterior, mostrando el mapa de contenidos del tema (figura } \\
\text { 3), explicando los principios que lo articulan. Se trata de hacer } \\
\text { a los alumnos partícipes del mapa de contenidos desarrollado, } \\
\text { para que comprendan la jerarquía de los conceptos básicos } \\
\text { trabajados en el tema. Se trabajan tareas sobre distintas } \\
\text { situaciones y contextos. }\end{array}$ \\
\hline $15(2 \mathrm{~h})$ & $\begin{array}{l}\text { En la primera parte de la sesión vamos a comenzar repasando } \\
\text { lo anterior, es decir, el vocabulario matemático implícito en las } \\
\text { fracciones, poniendo en común las respuestas de los alumnos } \\
\text { del día anterior. } \\
\text { En la segunda parte de la sesión, vamos a comenzar haciendo } \\
\text { reflexionar a los alumnos mediante la siguiente pregunta: ¿Cómo } \\
\text { determinar si dos fracciones son equivalentes? Además de esto, } \\
\text { vamos a trabajar las } 4 \text { operaciones básicas con fracciones (suma, } \\
\text { resta, producto y cociente de fracciones). }\end{array}$ \\
\hline $16(1 \mathrm{~h})$ & $\begin{array}{l}\text { En esta sesión comenzamos repasando lo visto en la anterior } \\
\text { clase. A continuación, desarrollamos algunos ejercicios para } \\
\text { afianzar los contenidos sobre las operaciones básicas con } \\
\text { fracciones. }\end{array}$ \\
\hline
\end{tabular}

Ciclos de Mejora en el Aula (2020). Experiencias de Innovación Docente de la US Esta obra se distribuye con la licencia Creative Commons Reconocimiento-NoComercial-SinObraDerivada Internacional (CC BY-NC-ND 4.0.) 


\begin{tabular}{|l|l|}
\hline $17(2 \mathrm{~h})$ & $\begin{array}{l}\text { En esta sesión se hace un breve repaso, recordando las } \\
\text { actividades realizadas en la sesión anterior. El profesor realiza } \\
\text { preguntas sobre los principales contenidos para analizar el } \\
\text { grado de comprensión de los alumnos (Orden de números } \\
\text { racionales y expresión decimal de un número racional). Además, } \\
\text { se resuelven las posibles dudas planteadas por los discentes } \\
\text { sobre el contenido teórico hasta ahora expuesto. }\end{array}$ \\
\hline $18(1 \mathrm{~h})$ & $\begin{array}{l}\text { En esta sesión desarrollamos algunos ejercicios para afianzar } \\
\text { contenidos. }\end{array}$ \\
\hline $19(2 \mathrm{~h})$ & $\begin{array}{l}\text { En esta sesión el profesor reformula las ideas extraídas de } \\
\text { las sesiones anteriores, intentando destacar los conceptos } \\
\text { fundamentales aprendidos durante estas sesiones. Se recopilan } \\
\text { las principales conclusiones sobre la proyección del mapa de } \\
\text { contenidos, para discutir con los estudiantes si consideran } \\
\text { necesaria alguna modificación o ajuste del mismo. }\end{array}$ \\
\hline $20(1 \mathrm{~h})$ & $\begin{array}{l}\text { Se les entrega el cuestionario final, cuestionario con las } \\
\text { mismas preguntas que en la primera sesión del CIMA. Se evalúa } \\
\text { positivamente la participación y se les recomienda usar el } \\
\text { mismo pseudónimo que para el cuestionario inicial, para poder } \\
\text { llevar a cabo su evolución. }\end{array}$ \\
\hline
\end{tabular}

\section{Aplicación del CIMA}

El inicio del CIMA coincide con el inicio de la asignatura y del curso. Hemos contado con una dedicación de 10 semanas. En el transcurso del CIMA hemos vivido un cambio por la situación de la pandemia en la que nos encontramos inmersos, pasando a la no presencialidad en las aulas. Este cambio no nos ha supuesto mucho desbarajuste en la programación pues lo habíamos barajado en el diseño. El comienzo de este escenario en el que $2 / 3$ de la clase se encontraba online y $1 / 3$ de forma presencial fue agotador, pues requería de estar atendiendo a las dudas de los presentes más las dudas de los que se encontraban en casa mediante el chat. Y a esto había que sumarle los fallos de la tecnología (cámara, micro, ...) para retransmitir la clase. Poco a poco todo se fue ajustando, y ya cuando todo parecía que iba bien, cambiamos de escenario, pasando a la no presencialidad total. Algo significativo en esto ha sido que, cuando los alumnos se encontraban en la presencialidad

Ciclos de Mejora en el Aula (2020). Experiencias de Innovación Docente de la US Esta obra se distribuye con la licencia Creative Commons 
del 33\%, asistían casi todos (tanto virtual como presencialmente), sin embargo, ahora en el escenario virtual 100\% no se ha reducido tanto la participación, pero sí la asistencia.

Un aspecto a destacar del CIMA, ha sido la observación de las ventajas que tenía el comenzar repasando lo impartido en la clase anterior, pues les anima a preguntar dudas si no lo habían entendido. Esto ya lo puse en práctica el curso pasado y me vuelve a dar resultado en este. Con respecto a la participación en clase, como constantemente planteamos preguntas y problemas no me puedo quejar, los alumnos están participando. En un par de ocasiones, alguna de las cuestiones que estaban diseñadas no alcanzaron el objetivo planteado y tuve que formular nuevas preguntas para su mejor entendimiento.

En este trabajo abarcamos tres módulos, aunque a día de hoy aún me encuentro impartiendo el tercer módulo. En cada uno de ellos, tanto al inicio como al final, he presentado el mapa de contenidos. Al inicio para ubicar lo que vamos a trabajar y, al final, como recordatorio de lo aprendido. La secuencia de actividades se ha ido desarrollando según lo previsto. El único cambio a destacar ha sido la realización de parte del cuestionario final propuesto para el 11 diciembre, que lo hemos realizado el día 26 de noviembre para poder realizar la evaluación del ciclo de mejora. Este ha sido modificado, abarcando solo las cuestiones referentes a los dos primeros temas (Preguntas 1-8).

\section{Evaluación del diseño}

Con el objeto de conocer cómo funcionan las clases preparadas para este trabajo hemos ido desarrollando un diario de profesor, así como el cuestionario inicial y final que hemos pasado al alumnado para conocer su experiencia de aprendizaje con este modelo metodológico. Además,

Ciclos de Mejora en el Aula (2020). Experiencias de Innovación Docente de la US Esta obra se distribuye con la licencia Creative Commons 
tras el cuestionario final, les pedimos que comentaran sus impresiones sobre la asignatura, planteando aspectos que mantendrían y cambiarían de la asignatura y de su puesta en práctica, como búsqueda de algunas pinceladas que a mí me sirvieran para mejorar. Todo esto me ha ayudado a valorar, proponer y mirar de forma constructiva este CIMA.

\section{Evaluación del aprendizaje de los estudiantes. Escaleras de aprendizaje}

Para la evaluación del aprendizaje de los estudiantes durante el ciclo de mejora se van a utilizar las escaleras de aprendizaje (Porlán, 2017). Las escaleras de aprendizaje son una representación gráfica en las cuales se muestran los avances de los estudiantes durante el CIMA. Como hemos reflejado anteriormente, no se ha podido completar todo el análisis. Mostramos las escaleras pertenecientes a los dos primeros módulos.

En las escaleras se establecen distintos niveles de aprendizaje, proponiendo respuestas prototípicas a cada uno de ellos. Tras recoger los cuestionarios, se van haciendo corresponder cada respuesta dada en estos niveles y se pasa a calcular el porcentaje de alumnos que se encuentra en cada uno de ellos. El cuestionario inicial fue rellenado por 63 personas y el cuestionario final ha sido elaborado por 49 alumnos, por lo que me quedo con las escaleras de estos últimos. Este descenso en la asistencia no sabemos si ha sido debido a la dejadez producida por la impersonalidad que conlleva seguir las clases por webcam o si ha tenido que ver algo el transcurso de la asignatura o ambas cosas, ya que la asignatura es de primer curso del grado de Educación Primaria.

En las siguientes figuras se muestran las escaleras de aprendizaje para las preguntas estructurantes del módulo 1 y 2. En cada pregunta establecimos tres niveles de

Ciclos de Mejora en el Aula (2020). Experiencias de Innovación Docente de la US Esta obra se distribuye con la licencia Creative Commons 
aprendizaje. Cada escalón corresponde a uno de los niveles de aprendizaje evaluados. En todas y cada una de las cuestiones observamos una evolución positiva del aprendizaje general. En cada pregunta aparecen las iniciales: I correspondiente a cuestionario inicial; F: cuestionario final; $\mathrm{N}$ : nivel de aprendizaje alcanzado.

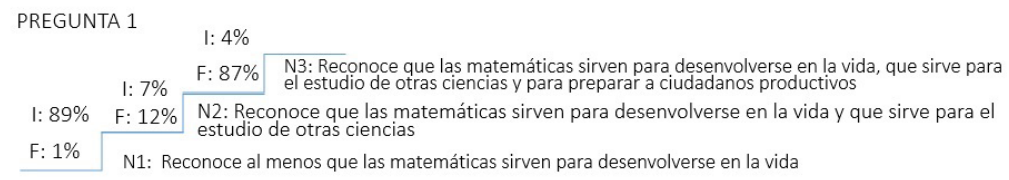

Figura 4. Escaleras de aprendizaje de las preguntas generales (Preguntas 1-3)

PREGUNTA 4
I: $2 \%$
F: 95\% N3: Distintos Sistemas de Numeración
I: $90 \%$ F: $5 \% \quad$ N2: Centenas, decenas y unidades
F: $0 \% \quad$ N1: No sabe/no contesta sobre distintos Sistemas Numeración

PREGUNTA 5
I: $5 \%$
I: $15 \%$ F: $9 5 \% \longdiv { \text { N3: Conoce propiedades del SND } }$
I: $80 \% \quad F: 5 \%$ N2: Nuestros números van de 10 en 10
F: $0 \% \quad N 1$ : Los números son infinitos

Figura 5. Escaleras de aprendizaje de las preguntas Módulo 1 (Preguntas 4-5)

Ciclos de Mejora en el Aula (2020). Experiencias de Innovación Docente de la US Esta obra se distribuye con la licencia Creative Commons 
F: 90\% N3: Conoce la definición y ejemplos de múltiplo y divisor

I: $50 \%$ F: $10 \% \quad$ N2: Conoce algún ejemplo de múltiplo y divisor

F: $0 \% \quad N 1$ : No sabe/no contesta sobre múltiplo y divisor

PREGUNTA 7

\section{I: $5 \%$}

F: 95\% N3: Conoce que es un número primo y cuáles son

$\begin{array}{lll}\text { I: } 70 \% & F: 5 \% & \text { N2: Conoce algún número primo }\end{array}$

F: $0 \% \quad$ N1: No sabe/No contesta sobre números primos

PREGUNTA 8
$\begin{array}{lllll} & \text { I: } 35 \% & \text { F: } 80 \% & \text { N3: Conoce mcd, } m \\ \text { I: } 60 \% & \text { F: } 20 \% & \text { N2: Sabe hallar mcd y mcm }\end{array}$
F: $0 \% \quad$ N1: No sabe/ no contesta sobre mcd y mcm

Figura 6. Escaleras de aprendizaje de las preguntas Módulo 2 (Preguntas 6-8)

Con respecto a las distintas escaleras de aprendizaje observamos como la mayoría de los estudiantes consiguió sin dificultad llegar al máximo nivel esperado. Algunos de los contenidos son tratados en niveles educativos inferiores, por lo que la mayoría de los estudiantes no partían de cero.

\section{Evaluación del diseño del CIMA}

Además del aprendizaje de nuestros estudiantes, nos gusta evaluar nuestra puesta en práctica. Para mí la puesta en práctica y la experiencia ha sido gratificante al observar que el alumnado logra avanzar en su aprendizaje. Como en cursos anteriores, debo comentar que la información que me aporta el cuestionario inicial es potente pues nos proporciona pistas para ver en qué partes hay que insistir más (Escudero-Domínguez, 2019). En este CIMA hemos conseguido ajustarnos a los tiempos propuestos, algo que siempre estaba de propuesta de mejora. Con respecto a

Ciclos de Mejora en el Aula (2020). Experiencias de Innovación Docente de la US Esta obra se distribuye con la licencia Creative Commons 
la evaluación de mi práctica por parte del alumnado, este curso no he usado el cuestionario que tengo elaborado de cursos anteriores, por falta de tiempo, sino que les indicamos que plasmaran sus impresiones mediante Google forms. En la última sesión, cuando ya cierre el CIMA completo, sí que tengo intención de pasarle el cuestionario que tengo elaborado de tipo escala Likert (Escudero-Domínguez, 2018).

Ciclos de Mejora en el Aula (2020). Experiencias de Innovación Docente de la US Esta obra se distribuye con la licencia Creative Commons 
Palabras clave: Matemáticas Específicas para Maestros, Grado en Primaria, Docencia universitaria, Experimentación docente universitaria

Keywords: Specific Mathematics for Teachers, Degree in Primary, University teaching, University teaching experimentation

\section{Referencias}

Bain K (2004). Lo que hacen los mejores profesores universitarios. Valencia: Publicaciones Universidad de Valencia

Carrillo, J., Climent, N., Gorgorió, N., Prat, M. y Rojas, F. (2008). Análisis de secuencias de aprendizaje matemático desde la perspectiva de la gestión de la participación. Enseñanza de las Ciencias, 26(1), 67-76.

Escudero-Domínguez, A. (2018). Aprendiendo a enseñar Didáctica de las Matemáticas en el Grado de Educación Primaria. Monográfico Jornadas de Formación e Innovación docente del Profesorado 1, pp. 216-229. DOI.: http:// dx.doi.org/10.12795/JDU.2018.i01.12 Pp.: 216-229

Escudero-Domínguez, A. (2019). Ciclo de Mejora en un aula de Asesoramiento Curricular y Competencias Básicas en Lengua, Literatura y Matemáticas. En E. Navarro-Medina y R. Porlán (Eds), Ciclos de Mejora en el aula, Año 2019: Experiencias de innovación docente de la Universidad de Sevilla (pp. 1907-1928). DOI.: http://dx.doi. org/10.12795/9788447221912.084

Finkel, D. (2008). Dar clase con la boca cerrada. Valencia: Universitat de Valencia.

Porlán, R. (Coord.). (2017). Enseñanza universitaria. Cómo mejorarla. Madrid: Ediciones Morata.

Tous, C.B. (2019). Entender la Bioquímica en Enfermería partiendo de casos problemas. En E. Navarro-Medina y R. Porlán (Eds), Ciclos de Mejora en el aula, Año 2019: Experiencias de innovación docente de la Universidad de Sevilla, pp. 863-885. DOl.: http://dx.doi. org/10.12795/9788447221912.038

Ciclos de Mejora en el Aula (2020). Experiencias de Innovación Docente de la US Esta obra se distribuye con la licencia Creative Commons 